\title{
VERS UNE POST-HUMANITE? ELEMENTS POUR UN DISCERNEMENT
}

Towards a Post-human Society ? Discerning Elements

François Euvé *

RÉSUMÉ : Le projet moderne de transformation technique de la nature s'étend désormais à l'humain. Pour comprendre ce qui se joue, il faut revenir au modèle mécanique au fondement de la cosmovision moderne. Ce modèle s'étend à l'esprit comme le montre l'exemple de l'intelligence artificielle. La machine est-elle conçue à l'image de l'homme, ou est-ce l'inverse ? Il faut donc réfléchir à la notion d'image, puisque la personne humaine est créée " à l'image de Dieu », afin de faire apparaître la dimension relationnelle de l'humain. La pensée de Teilhard de Chardin, qui invente la notion $\mathrm{d}^{\prime}$ " ultra-humain » peut aider au discernement. La transformation technique de l'humain doit être au service d'une plus grande communion entre les personnes.

MOTS-CLÉS: Image. Intelligence artificielle. Relation. Teilhard de Chardin. Transhumanisme.

\begin{abstract}
The modern project of technical transformation of nature now extends to the human person. To understand what is at stake, we must return to the mechanical model at the foundation of modern cosmovision. This model is extended to the mind as shown by the example of artificial intelligence. Is the machine designed in the image of the human being, or is it the other way around?
\end{abstract}

* Centre Sèvres-Facultés jésuites de Paris, Paris, France. 
Thus, it is necessary to think about the notion of image, since the human person is created "in the image of God", in order to make the relational dimension of the human being appear. The thought of Teilhard de Chardin, who invented the notion of the "ultra-human" can help with discernment. The technical transformation of the human being must be at the service of greater communion among people.

KEYWORDS: Image. Artificial Intelligence. Relation. Teilhard de Chardin. Transhumanism.

L

e projet de transformer la nature par l'usage de la technique est étroitement associé à la civilisation moderne. Depuis l'émergence d'une vision évolutive du vivant et de l'humain, ce projet s'est progressivement étendu à l'humanité elle-même. À partir du moment où l'on connaît le fonctionnement du corps humain, voire des facultés mentales, grâce à la connaissance du cerveau, n'est-il pas possible, et même souhaitable, de le modifier en vue de l'améliorer ? Une forme extrême et largement médiatisée de cette visée se rencontre dans le courant «transhumaniste », qui apparaît dans les années 1980. D'autres entreprises vont dans le sens d'une amélioration ou d'une " augmentation » (enhancement) de l'humain, sans se réclamer spécifiquement du transhumanisme.

Que recouvre ce projet qui, dans certaines de ses expressions, envisage le passage à une « post-humanité »? Une forme faible vise à améliorer la vie humaine en augmentant certaines de ses capacités (résistance aux maladies, vision ou audition plus précise et plus étendue, etc.). Une forme forte vise, plus radicalement, à s'affranchir de tout ce qui limite la vie humaine, les handicaps, la maladie et surtout la mort (HEFNER, 2009). Le rêve d'immortalité, aussi ancien que l'humanité comme en atteste le mythe de Gilgamesh, trouverait, grâce aux techniques nouvelles, un début de réalisation.

Le christianisme ne peut pas rester indifférent à ces différentes entreprises. Plusieurs raisons invitent le théologien à prendre au sérieux les débats qui $\mathrm{s}^{\prime} \mathrm{y}$ rapportent, sans se laisser polariser par les formes les plus extrêmes qui paraissent souvent délirantes. La première raison est que le projet moderne de transformation de la nature est né dans un environnement chrétien et, comme chez Francis Bacon (1561-1626), a trouvé ses premiers arguments dans la théologie chrétienne (FUDPUCKER, 1984; BURDETT, 2011, p. 20-25). Une deuxième raison est que des défenseurs du transhumanisme affirment qu'ils poursuivent le même objectif que les religions, mais par le seul usage d'une technique humaine, sans recourir à des instances surnaturelles. Il s'agirait d'une sorte de religion sans Dieu, d'une " post-religion », qui accomplirait la mission des religions en se débarrassant $\mathrm{du}$ bagage " mythologique » qui les encombre. Une troisième raison est le rapport à la mort. Le christianisme confesse que le Christ est « vain- 
queur de la mort ». Est-ce dans le même sens que ce que proposent les techniques d'immortalité ? Quelle fonction a la mort (plus largement, la fragilité de la « chair») dans une anthropologie chrétienne ?

Globalement, c'est la question de l'humain qui est posée. Jusqu'où aller dans l'usage de la technique appliquée à l'humain ? Qu'en est-il de la différence entre l'homme et la machine ? Plus radicalement, y a-t-il une " nature » humaine immuable ou l'homme est-il " a work in progress » (RIZZUTO, 2012, p. 569) ? Comment le discours chrétien sur l'homme peut-il rester crédible dans un contexte marqué par de spectaculaires développements technologiques ? Un discernement doit être opéré, auquel voudrait contribuer cet article.

Pour s'y repérer, il sera utile de commencer par quelques éléments historiques, afin de relever quelles sont les composantes de ce projet de transformation de l'humain. Une attention particulière sera ensuite donnée au domaine des sciences cognitives qui inspirent les développements de l'intelligence artificielle. C'est là où l'on voit le mieux la relation entre l'humain (la pensée) et la machine (l'ordinateur). Ce rapport en miroir (à l'idée que la machine simule l'humain se substitue celle que l'humain doit se conformer à la machine) invite à s'intéresser à la notion d' " image », centrale dans l'anthropologie chrétienne. Cela permettra de dégager l'importance de la dimension relationnelle de l'humain. Les entreprises transhumanistes apparaissent souvent comme des projets qui proposent « un individualisme libéral à tous points de vue, tant économique que social, tant politique que moral » (DAMOUR, 2015 , p. 38), allant jusqu'à valoriser « la puissance de l'individu comme unique centre de la morale »(DAMOUR, 2018, p. 152), rejetant, comme chez Max More, toute forme $d^{\prime}$ '« altruisme sacrificiel » (GOFFI, 2011, p. 30). Par contraste, la tradition chrétienne envisage le salut sous la forme d'une communauté de service et de partage. Cela pourra être illustré par une étude de la notion d' « ultra-humain » chez Teilhard de Chardin. Ce penseur chrétien est choisi parce quecar certains commentateurs en font un précurseur du transhumanisme. Il semble pourtant qu'en dépit de certaines ambiguïtés (une confiance excessive dans la technique), la réflexion teilhardienne puisse nous fournir des éléments de discernement.

\section{Un héritage historique}

Nick Bostrom, l'un des principaux acteurs du courant transhumaniste, le présente comme l'héritier d'une histoire longue (BOSTROM, 2005). Il ne serait que l'accomplissement du projet moderne, formulé par Francis Bacon, René Descartes ou Nicolas de Condorcet. Au sens large, on peut lui donner raison. De fait, c'est avec la modernité que la connaissance scientifique s'associe étroitement à une technique de transformation de la 
nature. Cette activité traditionnelle de l'humanité prend une figure nouvelle au début des temps modernes lorsqu'elle se trouve étroitement associée à une nouvelle connaissance scientifique du monde, davantage appuyée sur la rigueur démonstrative des mathématiques. Comme l'a fait remarquer Jean Ladrière, la science moderne a cette particularité de viser non plus la contemplation d'une nature ordonnée, mais sa transformation : " la science est un mode d'appréhension de la réalité qui relève essentiellement non de la vision, mais de l'action » (LADRIÈRE, 1977, p. 15). Connaître ne signifie plus tant lire le livre de la nature que de l'écrire à nouveau.

Quatre remarques peuvent être apportées à propos de cette nouvelle manière de se rapporter au monde.

La première est inspirée du célèbre ouvrage de l'historien-philosophe Alexandre Koyré, Du monde clos à l'univers infini (1962). L'instauration de la science moderne coïncide avec la découverte de nouveaux mondes. L'univers prend des dimensions sans commune mesure avec ce que connaissait l'homme des temps anciens. Les frontières du cosmos sont repoussées à l'infini. L'idée se fait jour que les limitations auxquelles l'humanité semble soumise depuis toujours (par « nature ») ne seraient pas définitives et qu'il serait donc possible de les transgresser.

La deuxième remarque tient au statut de l'humain. Selon la célèbre expression de Descartes, il est invité à se faire " comme maître et possesseur de la nature " (Discours de la méthode, sixième partie). La séparation est très nette entre l'ordre humain qui est celui de la liberté et l'ordre naturel qui est celui du déterminisme.

La troisième remarque est liée à la précédente. Le modèle selon lequel la nature est abordée est un modèle mécanique. Robert Boyle (1627-1691) est fasciné par l'horloge astronomique de Strasbourg (HOOYKAS, 1997). Le fonctionnement de cette machine conçue et réalisée par l'homme reproduit précisément le fonctionnement du ciel. L'univers ne serait-il pas une machine ? Le monde n'est plus compris selon un paradigme biologique (un grand « organisme») mais selon un paradigme mécanique.

La quatrième remarque aborde la résonance théologique. Le modèle mécanique est défendu par certains, comme Robert Boyle, par opposition au panthéisme associé au modèle organique. Comme l'écrit Newton dans le Scholie général qui achève les Principia, Dieu n'est pas « l'âme du monde » mais son " seigneur » (dominus). Il se distingue radicalement du monde. Comment mieux rendre sensible cette affirmation théologique, sinon par l'image de l'ingénieur qui conçoit, fabrique et entretient sa machine ? Inspiré par Newton, Voltaire parlera de Dieu comme l'« horloger » d'un monde horloge.

La nouveauté vient de l'émergence d'une vision évolutive du vivant qui inscrit davantage l'humain au sein d'un grand ensemble et qui rapproche 
aussi le monde vivant du monde physique. Les frontières classiques (inerte/ vivant/humain) commencent à se brouiller. L'idée d'une transformation de la nature peut s'étendre à l'humain.

Ce n'est pas fortuit que l'un des premiers emplois du mot «transhumanisme » se rencontre chez le biologiste Julian Huxley (1887-1975), petit-fils de Thomas Huxley, le défenseur de Darwin. Dans un texte de 1957, il avance l'idée d'une transformation de l'humain. L'homme est « l'agent qui a pour tâche de réaliser son potentiel inhérent aussi pleinement que possible. [...] Qu'il le veuille ou non, qu'il sache ou non ce qu'il fait, c'est en fait lui qui détermine l'orientation future de l'évolution sur cette terre » (HUXLEY, 1957).

Cette transformation de l'humain serait donc cohérente avec le grand mouvement de l'évolution. Mais il faut souligner une double nouveauté. Alors que l'évolution « naturelle » est un processus aléatoire, l'évolution nouvelle est un processus rationnellement dirigé. Au lieu d'être dépendant des hasards de la nature (modification du climat, événements fortuits), l'homme, grâce à ses connaissances scientifiques, peut acquérir la maîtrise des mécanismes. Par ailleurs, le tempo s'accélère. Alors que l'évolution antérieure supposait de très longues durées, il est possible de la rendre plus rapide.

Nous avons affaire à une conception progressiste de l'évolution. Pour Huxley, comme pour Pierre Teilhard de Chardin (1881-1955), sur qui nous reviendrons, il y a une cohérence dans l'histoire évolutive : la montée vers des organismes de plus en plus complexes. C'est cela qui donne un statut particulier à l'humain, et prépare le retournement qui s'opère sous nos yeux.

A-t-on affaire à une simple transformation de l'humanité, améliorant telle ou telle de ses caractéristiques, ou se dirige-t-on vers un stade ultérieur, une "post-humanité »? Comme suggéré plus haut, il existe différentes versions du transhumanisme, même si on qualifie habituellement de ce terme les formes les plus fortes, en particulier celle qui voudrait rendre l'humain immortel.

Cette version forte n'est pas sans résonance religieuse, même si, parmi ses défenseurs, la plupart se démarquent des religions traditionnelles et revendiquent clairement leur athéisme. On peut parler d'une « idéologie athée, mais religieuse sur le plan fonctionnel » (DAMOUR, 2017, p. 54), au sens où elle prend en charge les questions portées traditionnellement par les religions comme le salut, la mort ou le sens de l'histoire. Il semblerait que la technique " réalise le rêve immémorial des mythes religieux " (GOFFI, 2011, p. 21). Dominique Lecourt relève la présence d'un « vocabulaire du salut et de la vie éternelle » dans les textes des fondateurs de l'intelligence artificielle (LECOURT, 2011, p. 57). Les résonances peuvent être explicites ou plus cachées. Il n'est pas fortuit que Julian Huxley intitule son essai « Dans des bouteilles neuves pour un vin nouveau », allusion transparente 
à la phrase évangélique : «à vin nouveau outres neuves », le christianisme étant identifié à la religion de la nouveauté. En lien avec son emploi du mot "transhumanisme », William D. Lighthall (1857-1954) renvoie à la première épître aux Corinthiens : "ce que l'œil n'a pas vu, ce que l'oreille n'a pas entendu, et ce qui n'est pas entré dans la compréhension de l'homme » $(2,9)$, ce qui évoque sans doute l'enlèvement de Paul «jusqu'au paradis » où il entendit « des paroles inexprimables qu'il n'est pas permis à l'homme de redire » (2 Co 12,4). Ce passage est cité par Dante dans les vers du Paradis où il emploie le verbe " transumanar » qui a dû inspirer Lighthall (HARRISON, 2015, p. 466-467).

Parler de " dépassement » («trans ») ne peut pas ne pas évoquer ce qui transcende la condition humaine et qui oriente le regard vers le divin. Ce projet s'inscrirait-il dans l'aspiration de l'humanité à s'élever vers la divinité ? Les mythes antiques condamnent cette aspiration en plaçant une frontière infranchissable entre deux mondes, la «terre » et le « ciel ». Mais il faut réaliser que le christianisme, selon la doctrine de l'incarnation, rapproche considérablement l'humain du divin, puisqu'une même personne, Jésus-Christ porte en elle les deux natures. Le dogme souligne l'absence de confusion, mais laisse entendre que l'humain pourra partager la nature divine (« divinisation » ou " théôsis »), accédant à l'immortalité par la résurrection. Les miracles de Jésus (la guérison de l'aveugle-né, Jn 9) manifestent qu'aucune fatalité ne pèse sur la condition humaine.

Ce n'est pas fortuit que le projet transhumaniste, au même titre d'ailleurs que la science et la technique modernes, soit apparu dans un environnement culturel marqué par le christianisme. Cela rend le discernement délicat. Le christianisme ne reprend pas purement et simplement l'idée d'une "nature » intangible, puisque la nature humaine est destinée à être transformée à l'image du divin (HEFNER, 2009). Tout " naturalisme » est étranger au christianisme (GAGNON, 2012, p. 401). Il ne s'agit pas avant tout de " préserver » ou de " conserver » la nature mais de la "soumettre » et de la « dominer » $(\mathrm{Gn} 1,27)$ pour qu'elle corresponde toujours davantage au dessein créateur de Dieu. Cela dit, le christianisme ne valide pas non plus, à l'inverse, l'idée d'une humanité qui pourrait se transformer par ses propres forces pour atteindre l'état divin. La communion avec Dieu est un don reçu de Dieu lui-même. Nous reviendrons sur ce point essentiel.

\section{L'esprit mécanisé}

Avant de reprendre l'aspect théologique, il sera utile de revenir sur le modèle mécanique. Appliqué d'abord avec succès au monde physique, puis au monde vivant, peut-il aussi s'étendre au fonctionnement de l'esprit humain ? La machine peut-elle nous aider à comprendre comment nous 
raisonnons et, par conséquent, améliorer notre capacité de raisonnement ? Peut-on envisager de remplacer le cerveau par une machine ? Toutes ces questions font l'objet de grands débats du milieu du $20^{\mathrm{e}}$ siècle, au moment où l'on invente l'ordinateur (Alan Turing et John von Neumann), où l'on développe la théorie de l'information (Claude Shannon), où l'on découvre la structure du cerveau et les caractéristiques des neurones. Il semble que l'on puisse décrire le cerveau comme une " machine à traiter de l'information », ce qui le rapproche de l'ordinateur.

Les pionniers de l'informatique ont exprimé clairement leur conception des nouvelles machines. Loin d'être simplement des outils pour nous aider dans notre vie quotidienne ou notre travail, ce sont des modèles qui nous aident à mieux comprendre ce que nous sommes. L'ordinateur doit permettre d'acquérir « une compréhension plus profonde du comportement humain »(SIMON, 1974, p. 38).

Cela relève du domaine des sciences cognitives. Comme l'écrit Laurence Devillers, " L'objectif des sciences cognitives et de l'intelligence artificielle est à la fois de comprendre les mécanismes du raisonnement humain et du fonctionnement du cerveau à l'aide de simulation des processus cognitifs sur ordinateur, mais également de créer des machines artificiellement intelligentes pour nous assister avec lesquelles nous pouvons communiquer » (DEVILLERS, 2017, p. 70). L'objectif est donc double : compréhension et fabrication. Le premier se dédouble à son tour : comprendre le raisonnement et comprendre le fonctionnement du cerveau. Le développement des sciences cognitives suit de très près celui des neurosciences. C'est la découverte du neurone qui permet d'imaginer que l'on va construire des machines électriques qui en reproduisent le comportement. On pense voir " une analogie entre le fonctionnement du système nerveux et celui des machines bâties comme des réseaux électroniques» (MALDAMÉ, 2002, p. 52).

L'interrogation sur le propre de l'homme attire l'attention sur le langage et, plus précisément, sur le langage bien formé, le raisonnement logique. Les règles de la logique sont forgées au moins depuis l'Antiquité grecque. Une étape est franchie lorsque le formalisme logique est rapproché du formalisme mathématique (George Boole, 1815-1864). Le fonctionnement de l'esprit doit pouvoir être décrit à l'aide du langage mathématique.

Depuis Galilée, c'était déjà le cas du fonctionnement du monde. Les phénomènes physiques obéissent à des lois que l'on peut exprimer à l'aide des mathématiques. L'extension se fait progressivement au monde vivant. Un seuil symbolique est franchi lorsque James Watson et Francis Crick établissent en 1953 la structure géométrique de la molécule d'ADN, ce qui explique le mécanisme de la reproduction des organismes vivants.

Ce qui s'applique bien au monde physique et désormais au monde vivant peut-il s'appliquer aussi au monde de l'«esprit», en particulier si on le 
comprend surtout à travers le fonctionnement de l'intelligence humaine. La pensée pourrait-elle se ramener à un calcul, comme l'affirmait Thomas Hobbes ? Elle relèverait alors « de l'ordre du mécanique » (DUPUY, 2000, p. 162). Le rapprochement entre logique et mathématique va dans cette direction. Il ne restait qu'à inventer des machines qui puissent matérialiser cette analogie. La numération binaire, inventée par Leibniz, est commune à la logique (vrai/faux) et aux circuits électriques (ouvert/fermé) auxquels on assimile le fonctionnement des neurones. On peut définir l'intelligence artificielle comme " une discipline basée sur l'hypothèse fondamentale selon laquelle les processus de pensée sont mécanisables et peuvent être simulés sur ordinateur » (HOUDÉ, 1998, p. 147). Si l'on arrive à décrire précisément le fonctionnement de l'intelligence, on doit pouvoir construire une machine pour la simuler.

Il ne s'agit pas seulement de machines logiques qui appliquent rigoureusement des règles définies pour aboutir à un raisonnement valide. On parlera de machine « intelligente » lorsqu'elle devient capable d'adaptation à des situations nouvelles : "Un système intelligent est un système capable de s'adapter, de façon autonome, à des situations non pré-programmées » (LE MOIGNE, 1986, p. 46).

La frontière entre l'homme et la machine devient de plus en plus poreuse. Qui est l'image de l'autre? «La fragilité des frontières entre intelligence et intellect, cerveau et intellect, machine et intellect, intelligence (naturelle) et intelligence (artificielle), est devenue si manifeste qu'elle interdit tout partage assuré entre le biologique, le mécanique et le symbolique » (MALABOU, 2017, p. 24). On parle d'ordinateur " intelligent ", c'est-à-dire possédant des propriétés qui le rapproche de l'humain. Jean-Pierre Dupuy suggère que ce serait plutôt l'inverse : " La cybernétique, ce n'est pas l'anthropomorphisation de la machine, c'est d'abord la mécanisation de l'humain » (DUPUY, 2000, p. 163). L'accomplissement de l'humanité serait une sorte de machine idéale, raisonnant selon une logique parfaite.

\section{Le mécanisme de l'idolâtrie}

S'il y a bien «mécanisation de l'humain ", c'est que la technique, qui se fonde comme on l'a vu sur un modèle mécanique, affecte directement l'humain en tant qu'humain. Il n'est pas fortuit que le transhumanisme accorde une valeur centrale à la technologie. Le premier article de la « Déclaration transhumaniste » débute par cette affirmation : "L'humanité sera radicalement modifiée à l'avenir par la technologie » (DAMOUR, 2015, p. 156). Cet idéal se fonde "sur une reprise de l'évolution, une façon de perfectionner et de transcender la race humaine $[. .$.$] non à l'aide des mutations biologiques mais à l'aide de$ la science et de la technologie »(GAGNON, 2012, p. 393). 
Mais la technique ne connaît que des « choses » et non des «personnes". Elle réifie ce qu'elle affecte. Transformer techniquement l'humain, n'est-ce pas le réduire à l'état d'une chose matérielle ? Des partisans du transhumanisme ne font pas de distinction essentielle entre l'homme et le robot, autrement dit, entre l'homme et la machine, aussi « intelligente » soit-elle. Certains voudraient conférer aux robots le statut juridique de " personne ». Pour Norbert Wiener, la personne humaine et l'ordinateur sont deux variétés d'un même genre, les machines informationnelles. Il est clair qu'il ne s'agit pas d'une machine au sens banal, " matériel ", d'un moteur ou d'un engin simplement mécanique. La dimension essentielle est l'information qui paraît « dématérialiser ». Mais est-ce si différent ? Le projet transhumaniste reste à l'intérieur de la conception mécaniste du vivant (TESTART, 2018, p. 11).

La fascination pour la machine, surtout lorsqu'elle est « intelligente », risque d'entraîner un retrait de l'humain dans sa capacité de décision : «À force de se faire remplacer par les machines, n'assisterons-nous pas à une prise de pouvoir passive des machines, par simple démission des hommes ? » (GANASCIA, 2017, p. 57). "Plus nous dépendons de la technologie, plus nous risquons de perdre les capacités qui nous permettent de prendre des décisions sans cette technologie »(HERZFELD, 2002, p. 78). L'humanité gardera-t-elle la maîtrise de ses objets ou sera-t-elle dominée par eux ? Des spécialistes du domaine se veulent rassurants : «Les machines ne sont pas en mesure à l'heure actuelle de concurrencer les hommes » (DEVILLERS, 2017, p. 78). Mais qu'en sera-t-il demain?

Cela évoque le principe de ce que l'historien Franck Damour appelle le " cercle idolâtrique » : pour se sauver, l'homme doit devenir une machine qu'il a lui-même construite (DAMOUR, 2015, p. 131). L'idolâtrie des objets techniques avait été dénoncée par le philosophe français Jacques Ellul (ELLUL, 1954). Il analysait le retournement qui s'opère lorsque des instruments qui sont censés nous rendre indépendants de la nature en nous assurant une puissance sur elle, deviennent à leur tour des occasions d'asservissement. Nous nous mettons à dépendre d'eux, comme si nous étions soumis à leur pouvoir. À la différence de l'objet esthétique, qui a une valeur par lui-même, l'objet technique n'a de valeur qu'utilitaire, c'est-à-dire référée à celui qui l'utilise. Il peut alors être investi de valeur "fantasmée ", projection du rêve de son concepteur.

Dans le champ biblique, l'idolâtrie consiste à attribuer une caractéristique divine à un objet fabriqué par l'homme. Les chapitres 13 et 14 du livre de la Sagesse décrit admirablement le mécanisme de l'idolâtrie. Pour s'assurer la santé, la protection dans les voyages et même la vie, l'homme s'en remet à un objet qu'il a fabriqué et à qui il confère un pouvoir divin. Par peur de la mort, l'homme se fabrique des idoles. Il leur transfère l'immortalité qu'il aimerait posséder. Mais, en fin de compte, il se retrouve esclave de l'objet par lequel il pensait s'assurer un pouvoir sur les choses. 
La Bible n'a pas de mal à dénoncer l'absurdité de la situation : comment un objet qui dépend de l'homme qui l'a conçu et réalisé peut-il revêtir des qualités suprahumaines ? L'idole a beau avoir des caractéristiques humaines, elle s'avère plutôt infrahumaine : " elles ont une bouche et ne parlent pas » (Ps 115,5). On pourrait ajouter à l'observation du psalmiste la remarque qu'il serait possible de faire "parler » des objets fabriqués par l'homme. C'était le cas de statues dans l'Antiquité, d'automates du $18^{e}$ siècle et, bien sûr, d'ordinateurs actuels capables de " dialoguer " avec des personnes humaines. Mais, dans tous les cas, il s'agit de constructions artificielles qui ne permettent pas de considérer ces échanges comme d'authentiques dialogues.

\section{Quelle image?}

L'idole est la perversion de l'image. Que la machine soit "à l'image » de l'humain (lorsque l'ordinateur devient « intelligent») ne signifie pas nécessairement qu'elle devienne par le fait-même une idole. La frontière est ténue, comme l'attestent les nombreuses tentatives de réaliser des artefacts à l'image de l'homme. Un exemple caractéristique est celui du " Golem ». Son «moulage » rappelle fortement la création du premier humain selon le second récit de création dans la Genèse. L'analogie ne peut pas manquer d'être établie entre la réalisation de l'humain par un Dieu qui lui donne " souffle de vie » (c'est-à-dire capacité d'autonomie) et la réalisation par cet humain d'une machine à laquelle il confère une semblable capacité d'autonomie. L'inspiration biblique du Golem est transparente (BRETON, 1995 , p. 82). La notion d'image engage une certaine distance, une non-

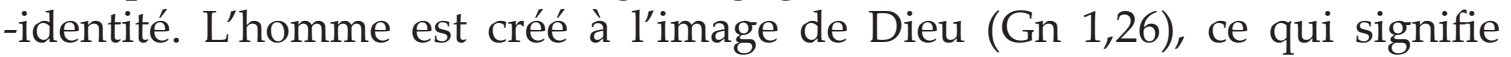
qu'il garde une certaine dépendance à l'égard de son Créateur. Il nous faut préciser le sens à donner au mot «image».

Schématiquement, trois interprétations ont été proposées pour rendre compte de cette notion (HERZFELD, 2002). La première en fait une propriété intrinsèque à l'humain qui reflète la puissance divine, la différence entre Créateur et créature. Elle met l'accent sur la raison $(\log o s)$ et, dans un héritage platonicien, distingue nettement entre l'esprit (raisonnable) et le corps (siège des passions qui sont communes à l'homme et à l'animal). Par son esprit, l'homme est capable de dominer ses passions, d'y mettre de l'ordre, comme Dieu avait, au commencement du temps, ordonné le chaos primordial.

Une deuxième interprétation est plus fonctionnelle. Rejetant une lecture qu'il trouve trop intellectualiste, Gerhard von Rad propose de voir l'homme comme le représentant de Dieu dans le monde. C'est par son agir ou sa puissance (en particulier lorsqu'il transforme les choses) que l'homme se 
reconnaît à l'image de Dieu. Ce trait a été mis en valeur par les initiateurs de la modernité, en particulier Francis Bacon. L'action humaine qui transforme la nature au profit de l'humanité s'inscrit dans le dessein créateur de Dieu. Par là, l'homme accomplit la mission que lui a confiée le Créateur.

Une troisième interprétation est relationnelle. Karl Barth rapproche la création à l'image de Dieu de la relation fondatrice qui est celle de l'homme et de la femme. En outre, si l'on reconnaît la dimension trinitaire de l'action créatrice, on ne l'oubliera pas lorsqu'il sera question de l'image. Ce qui constitue l'humain n'est pas une puissance solitaire, mais l'inscription dans une relation d'amour.

Cette dernière interprétation ouvre une perspective relationnelle. La réflexion sur l'intelligence artificielle peut être l'occasion de mieux percevoir, par contraste, en quoi l'humain est essentiellement un être de relation. "Par contraste " car, comme l'a montré Jean-Pierre Dupuy dans sa lecture de la cybernétique et des sciences cognitives, qui sont à l'arrière-plan de l'intelligence artificielle, on a affaire à un « processus sans sujet » (DUPUY, 1999, p. 112).

Les développements classiques mettent, pour une part à juste titre, l'accent sur la dimension individuelle de la personne humaine douée d'autonomie et de liberté. Le processus éducatif vise à former cette individualité, en développant en particulier l'intelligence du jeune enfant. Le fait de comprendre le fonctionnement du monde physique (et des sociétés humaines) permet de s'en rendre davantage indépendant. L'humanité admire les " grands hommes » qui ont su, par leur intelligence, dominer les situations et gouverner les sociétés. L'opinion estime davantage l'intelligence que la seule force physique, voire la beauté.

La question se pose inéluctablement lorsque les machines parviennent à dépasser les performances d'une personne humaine, aussi intelligente qu'elle soit. C'est déjà le cas dans le domaine des jeux. C'est de plus en plus le cas dans le domaine médical (les diagnostics établis par ordinateur peuvent être plus fiables que ceux des spécialistes humains). Cela peut être le cas dans le domaine judiciaire (établir des jugements équilibrés), financier (acheter ou vendre des titres), économique (gérer une entreprise), voire politique (prendre de bonnes décisions, en évitant le jeu des passions). Doit-on penser que les machines vont remplacer les humains dans tous ces domaines?

Le thème de la puissance n'est pas absent de la tradition biblique. Dans les évangiles synoptiques, les guérisons de Jésus sont présentées comme des «actes de puissance "(dunameis). À s'en tenir à ses seuls miracles, on pourrait se le représenter comme un «surhomme » dont les capacités dépassent celles de l'humain ordinaire. Mais est-ce bien là la pointe du message évangélique ? Sur la croix, Jésus refuse d'user de cette puissance pour s'affranchir de la mort. Par ailleurs, il rassemble des disciples pour 
continuer à faire ce qu'il fait, comme le montrent les récits des Actes des apôtres. L'accent est moins mis sur la puissance que sur le partage. C'est bien ce que manifeste le geste eucharistique : son corps est partagé entre les convives.

L'accomplissement de la puissance est dans le service. "Celui qui veut devenir grand, qu'il se fasse le serviteur de tous » (Mt 23,11). Ce n'est pas là simplement un message "spirituel », sans effet pratique. L'efficacité apparente d'une performance individuelle (l'ordinateur qui bat le champion d'échec) ne doit pas cacher le fait que l'intelligence partagée (ou collective) est souvent plus pertinente dans le long terme. Sans doute les systèmes « intelligents » ont-ils accumulé l'expérience de nombreux acteurs grâce au " big data », mais il ne s'agit que d'une synthèse d'expériences passées. Pour Noreen Herzfeld, « l'idée d'une intelligence individuelle est dépourvue de sens [...] l'intelligence n'a de sens que dans la rencontre » (HERZFELD, 2012b, p. 507).

Le fantasme d'une entité omnisciente qui décide à notre place hante l'humanité depuis toujours. Les organisations sociales, que ce soit les Etats ou les Eglises, ont une composante hiérarchique qui donne à certains une capacité de décision plus forte qu'à d'autres. Mais, là encore, le modèle évangélique, sans se positionner délibérément dans le champ politique, suggère une autre logique qui repose sur le service, c'est-à-dire l'ouverture à autrui.

\section{$5 \mathrm{Du}$ post-humain à l'ultra-humain}

L'idée de faire venir la personne de Teilhard de Chardin dans le cadre d'une réflexion sur le post-humain peut se soutenir si l'on remarque que son nom est parfois invoqué par ceux qui défendent l'idée d'une transition à venir de l'humanité vers un état « autre». Pour certains, il pourrait donner un visage chrétien aux entreprises du trans- ou du post-humanisme (STEINHART, 2008). Pour d'autres, critiques de ces courants, il serait, en association avec son ami Julian Huxley, un inspirateur ou un "prophète " du transhumanisme (LE DÉVÉDEC, 2015, p. 134). Quoi qu'il en soit, « peu de théologiens ont offert davantage d'ouvertures suggestives pour un dialogue constructif avec le transhumanisme que le jésuite français Pierre Teilhard de Chardin » (GRUMETT, 2011, p. 37).

Teilhard n'emploie pratiquement jamais le mot de «transhumain », familier pourtant à son ami Julian Huxley. En revanche, il forge celui d'« ultra-humain » qui représente à ses yeux l'accomplissement de l'humanité. La différence principale n'est pas tant la composante religieuse - on y reviendra - que la dimension collective ou sociale qui s'y attache. 
Pour Teilhard, on l'a rappelé en commençant, l'univers entier est en évolution continue, ce qui signifie que la création se poursuit. Cela concerne au premier chef l'être qui représente la pointe de l'évolution, à savoir la personne humaine. Son évolution n'est pas seulement biologique ou anatomique (celle-ci se poursuit mais lentement) ; elle est surtout culturelle et sociale. D'une part, par son action technique, l'homme transforme son environnement et se transforme lui-même. D'autre part, l'humanité développe de plus en plus d'interdépendances. Elle constitue sur la surface du globe, une " couche de pensée " que, par analogie avec la biosphère, Teilhard baptise « noosphère ».

Le tissu de plus en plus serré qui réunit les humains dessine le profil d'une entité nouvelle, une sorte d'unanimité humaine qui représente un « changement de phase ». L'humanité n'est plus la juxtaposition d'individus ou de sociétés isolées les unes des autres, mais constitue un vaste ensemble, une communion. Elle se "socialise ", c'est-à-dire qu'elle "se brasse, s'emmêle, et se lie plus étroitement chaque jour sur elle-même " (TEILHARD DE CHARDIN, 1963, p. 320). Cette insistance sur la dimension collective n'est pas antinomique du maintien de la singularité des personnes. Pour lui, " l'union différencie », et c'est en se connectant toujours davantage aux autres que la personne humaine « acquiert davantage d'être » (DELIO, 2012, p. 163).

Il existe pourtant de puissantes forces d'opposition, c'est-à-dire de dispersion. Le "serrage » de l'humanité, ne serait-ce que pour des raisons démographiques, est source de conflits. Qu'est-ce qui peut assurer que les puissances de division ne l'emporteront pas ? Placer tout son espoir dans les seules capacités humaines serait illusoire. Le regard de Teilhard prend sa source dans un acte de foi. Le rassemblement est potentiellement réalisé dans le corps du Christ. Dans la lettre aux Colossiens, Teilhard lit que le Christ a tout réconcilié en lui. Ce n'est pas seulement une espérance lointaine ou une vue " pieuse ». L'observation du monde atteste que le rassemblement est déjà à l'œuvre.

Il en est de même au niveau individuel. La mort semble une "barrière » infranchissable qui paraît ruiner à l'avance tout projet de croissance. Mais la dialectique teilhardienne joue aussi à ce niveau : autant il est légitime de lutter contre la maladie, voire utiliser les ressources de la science pour " améliorer » l'humain, autant vient le temps du renoncement, de l'abandon, de la remise de soi à autrui, tout spécialement à Celui qui a connu la mort mais l'a traversée.

La technique a donc valeur à ses yeux, car c'est la manière avec laquelle l'humanité poursuit l'œuvre créatrice de Dieu. Il faut seulement remarquer que le critère est essentiellement d'ordre social. L'enjeu principal n'est pas tant d'améliorer l'individu (ce que Teilhard n'exclut pas), que d'agir dans 
la perspective du rassemblement de l'humanité. Le progrès technique nous oblige à choisir entre « l'esprit prométhéen ou faustien : esprit d'autonomie et de solitude » et " l'esprit chrétien : esprit de service et de don », autrement dit, entre « l'esprit de force » et « l'esprit d'amour » (TEILHARD DE CHARDIN, 1959, p. 187).

Teilhard est trop conscient de l'ambivalence de la condition humaine et de la présence du mal dans le monde pour penser qu'à elle seule, la technique pourrait assurer le salut de l'humanité. S'il accorde une grande valeur à l'activité humaine (à l'encontre des courants spirituels qui la dévalorisent), il en reconnaît une plus grande à la passivité qui conduit à s'abandonner une fois que l'on a fait ce que l'on doit faire.

C'est là un point difficile dans la pensée teilhardienne : comment dire à la fois la valeur du travail humain et affirmer que cette valeur n'est pas absolue puisque le salut relève d'un don gratuit ? Teilhard est la cible de ceux qui, comme Jacques Ellul, assimilent la technique moderne à une entreprise de mécanisation de l'humain. Certains textes de lui manifestent certainement un optimisme excessif quant aux résultats à attendre d'une transformation technique de l'humain. Mais ces textes demandent à être équilibrés par d'autres qui mettent en avant la liberté de la personne qui ne doit pas être réduite " aux développements mécaniques d'une collectivité sans âme » (TEILHARD DE CHARDIN, 1965, p. 181).

Il est donc abusif d'identifier l'ultra-humain teilhardien au post-humain des transhumanistes. Les deux motifs principaux sont d'une part la dimension sociale de l'ultra-humain qui met en avant la solidarité de l'ensemble de l'humanité et d'autre part le fait que son atteinte ne résulte pas de la seule activité humaine. Pour Teilhard, comme pour la tradition chrétienne, la mort n'est ni une fatalité qui marquerait la fin de tout, ni une simple « maladie » qui pourrait disparaître un jour. Comme l'atteste la résurrection du Christ, la «barrière de la mort » peut être traversée mais cela ne résulte pas de la seule activité humaine. Cela reste un don reçu de Dieu.

\section{Eléments de discernement}

En conclusion, il est possible de prolonger la ligne teilhardienne, reprenant ce qui était dit précédemment sur le corps et ses limites. Les techniques actuelles permettent d'envisager la traversée de limites autrefois considérées comme "naturelles », c'est-à-dire données, voire imposées à l'homme. Ce n'est pas nouveau : les ballons puis les avions permettent de s'élever dans l'air, voire de quitter la gravitation terrestre. Les télescopes permettent de voir ce qui restait invisible à l'œil nu. Ce qui est nouveau est ce qui affecte le domaine de l'esprit, du mental ou de l'intelligence, 
ce qui semblait le plus spécifique de l'humain. L'idéal humain serait-il la super-intelligence, déconnectée de tout support organique (mais pas de tout support matériel) ?

Il est banal de dire que les progrès techniques n'apportent pas nécessairement une amélioration de la condition humaine. La dégradation de l'environnement, qui se manifeste en particulier dans la crise climatique, est le résultat de l'industrie humaine. Ce n'est pas que toute entreprise technique soit mauvaise ou dangereuse, même quand elle s'applique à l'humain. Il faut simplement prendre conscience de son ambivalence, aggravée par le caractère imprévisible de ses conséquences. On ne peut jamais savoir précisément à l'avance quelles seront les conséquences à long terme d'une technique donnée. Celui qui est formé à la physique mathématique risque de l'oublier, en pensant que sa méthode lui donne prise sur le temps. Mais la complexité des situations réelles introduit un horizon d'incertitude au delà duquel il serait imprudent de se prononcer.

Il ne s'agit pas de condamner la technique comme telle (surtout dans la mesure où l'on en profite!), mais d'évaluer les critères qui font qu'une technique est vraiment " humaine ». S'en remettre à la technique, surtout si l'on pense que son expression la plus aboutie se trouve dans les ordinateurs "intelligents », pourrait signifier s'en remettre à une instance anonyme, impersonnelle. On pourra objecter que, dans plusieurs domaines, les machines se sont avérées plus fiables que les humains (diagnostics médicaux, par exemple). Ce peut être une aide, mais aussi un danger, celui d'abdiquer sa capacité de décision, sa responsabilité. Celle-ci comporte une dimension collective : la décision engage une délibération, un échange. $C e$ qui nous fait humain, c'est la parole qui circule entre nous.

Dès les premiers projets d'amélioration de l'humain, le biologiste Jean Rostand affirmait qu'il n'y a qu'une façon pour l'humain de grandir : " c'est par la générosité, le dévouement, le don de soi » (TESTART, 2018, p. 181). On peut prolonger cette dernière idée en rappelant qu'une anthropologie chrétienne est profondément relationnelle. Elle propose comme horizon de salut la réconciliation universelle, la constitution d'une communion des sauvés, anticipée par l'Église, où Dieu sera «tout en tous » $(1$ Co 15,28). On retrouve l'inspiration teilhardienne.

L'entrée en relation de communion ou d'amour suppose deux conditions qui sont d'ailleurs en relation dialectique : l'existence de personnes libres à l'égard de toute dépendance qui les empêcherait de poser un vrai choix ; l'acceptation de sortir de soi au profit d'autrui, ce que l'Évangile appelle " perdre son âme ». Le paradoxe de l'amour, effectivement vécu par ceux qui vivent une authentique relation, est que l'âme " perdue » est redonnée par la relation. Comme ne le cesse de répéter Teilhard «l'union personnalise ». 
Cela ne plaide pas pour renoncer à toute action de " puissance ». Par la technique, l'homme acquiert davantage d'autonomie à l'égard des déterminismes naturels. Il n'est plus le jouet de forces obscures. C'est ainsi que l'on peut se représenter le grand récit de l'évolution comme une montée de complexité dans les organismes, et donc de conscience, c'est-à-dire de capacité de s'approprier son environnement tout en s'en distançant. Rien n'empêche de considérer l'homme qui transforme son environnement ou se transforme lui-même comme participant à l'amélioration de la condition humaine. Par son travail, y compris le plus technique, l'humanité participe au projet créateur de Dieu.

Mais ce projet ne pourra être atteint que par renoncement à la maîtrise totale sur les choses. C'est le piège de la puissance, surtout lorsqu'elle est détenue par des individus qui en usent à leur seul profit (ce que la tradition de l'Église appelle le péché d'orgueil où saint Augustin voyait la racine de tout péché). La proximité entre les entreprises d'amélioration ou d'augmentation de l'humain et les idéologies "libertariennes » qui valorisent exclusivement l'individu jusqu'à affirmer la valeur de l'égoïsme, pose question. À l'encontre d'une telle attitude, ne seront profitables que les techniques partagées par l'ensemble de l'humanité.

\section{Références}

BOSTROM, N. A history of transhumanist thought. Journal of Evolution and Technology, USA, v. 14, issue 1, Apr.il 2005.

BRETON, P. À l'image de l'Homme: du Golem aux créatures virtuelles. Paris: Seuil, 1995.

BURDETT, M. S. Contextualizing a Christian Perspective on Transcendance and Human Enhancement: Francis Bacon, N. F. Fedorov and Pierre Teilhard De Chardin. In: COLE- TURNER,E.. 2011. p. 19-36.,DAMOUR, F. La tentation transhumaniste. Paris: Salvator, 2015.

Le transhumanisme, une idée chrétienne devenue folle?. Etudes, Paris, p. 51-62, juil./août 2017.

. Le mouvement transhumaniste. Approches historiques d'une utopie technologique contemporaine. Vingtième siècle, Paris, n. 138, p. 143-156, 2018.

DELIO, I. Transhumanism or ultrahumanism? Teilhard de Chardin on technology, religion and evolution. Theology and Science, Berkeley , v. 10, issue 2, p. 153-166, 2012.

DEVILLERS, L. Des robots et des hommes: mythes, fantasmes et réalités. Paris: Plon, 2017.

DUPUY, J.-P. Aux origines des sciences cognitives. Paris: La Découverte, 1999. avr. 2000 .

. L'esprit mécanisé par lui-même. Le Débat, Paris, n. 109, p. 161-176, mar./ 
ELLUL J. La Technique, ou l'Enjeu du siècle. Paris: Armand Colin, 1954.

FUDPUCKER, W. Throught Christian Technology to Technological Christianity. In: MITCHAM, C.; GROTE, J. (Eds.). Theology and Technology: Essays in Christian Analysis and Exegesis. Lanham, [Md.]: University Press of America, 1984. p. 53-69,.

GAGNON, P. The Problem of Transhumanism in the Light of Philosophy and Theology: 2017-03-05. In: STUMP, J. B.; PADGETT, A. G. (Eds.). The Blackwell Companion to Science and Christianity. Chichester. West Sussex ; Malden, MA: Wiley-Blackwell, 2012.

GANASCIA, J.-G. Intelligence artificielle: vers une domination programmée. Paris: Le Cavalier Bleu, 2017. (Idées reçues).

GERACI, R. M. Cyborgs, Robots, and Eternal Avatars. In: HAAG, J. W.; PETERSON, G. R.; SPEZIO, M. L. (Eds.). The Routledge Companion to Religion and Science. London; New York: Routledge, 2012. p. 578-590.

GOFFI, J.-Y. Nature humaine et amélioration de l'être humain à la lumière du programme transhumaniste. Journal International de Bioéthique, Paris, v. 22, n. 3-4, p. 18-32, 2011.

Aux origines contemporaines du transhumanisme. Julian Huxley et Fereidoun M. Esfandiary. Ethique, politique, religions, Lyon, v. 6, n. 1, p. 17-32, 2015.

GRUMETT, D. Transformation and the end of Enhancement: Insights from Pierre Teilhard de Chardin. In: COLE-TURNER, R. (Ed.). Transhumanism and Transcendence: Christian Hope in an Age of Technological Enhancement. Washington, D.C.: Georgetown University Press, 2011.

HARRISON, P.; WOLYNIAK, J. The history of transhumanism. Notes and Queries, Oxford, July 2015.

HEFNER, P. The animals that aspires to be an angel: the challenge of transhumanism. Dialog, Dialog: A Journal Of Theology, St. Paul, v. 48, n. 2, p. 164-173, 2009.

HERZFELD, N. L. In Our Image: Artificial Intelligence and the Human Spirit. Augsburg Books, 2000.

Human-Directed Evolution. A Christian Perspective. In: HAAG, J. W.; PETERSON, G. R.; SPEZIO, M. L. (Eds.). The Routledge Companion to Religion and Science. London; New York: Routledge, 2012a. p. 591-6011,.

In Whose Image? Arficial Intelligence and the Imago Dei. In: STUMP, J. B.; PADGETT, A. G. (Eds.). The Blackwell Companion to Science and Christianity. Chichester, West Sussex; Malden, MA: Wiley-Blackwell, 2012b. p. 500-509.

HOOYKAAS, R. Robert Boyle: a Study in Science and Christian Belief. Ancaster, Ont.: Lanham, Md: Pascal Centre for Advanced Studies in Faith and Science, Redeemer College; University Press of America, 1997.

HOUDÉ, O. (Ed.). Vocabulaire de Sciences Cognitives: Neuroscience, Psychologie, Intelligence Artificielle, Linguistique et Philosophie. Paris: PUF, 1998.

HUXLEY, J. S. New bottles for new wine: essays. London: Chatto \& Windus, 1957.

LADRIÈRE J. Les Enjeux de la rationalité: le défi de la science et de la technologie aux culture. Paris: Aubier-Montaigne, 1977. 
LECOURT, D. Humain, posthumain. Paris: PUF, 2011.

LE DÉVÉDEC, N. La société de l'amélioration: la perfectibilité humaine des Lumières au transhumanisme. Montréal: Liber, 2015.

LE MOIGNE, J.-L. (Ed). Intelligence des mécanismes, mécanismes de l'intelligence: intelligence artificielle et sciences de la cognition. Paris: Fayard, 1986.

MALABOU C. Métamorphoses de l'intelligence: que faire de leur cerveau bleu? Paris: PUF, 2017.

MALDAMÉ, J.-M. Faut-il avoir peur des sciences cognitives? Etudes, Paris, v., n. p. 51-62, jJanv.ier 2002.

RIZZUTO, D.; FOST, J. Transhumanism and Cognitive Enhancement. In: HAAG, J. W.; PETERSON, G. R.; SPEZIO, M. L. (Eds.). The Routledge Companion to Religion and Science. London/New York: Routledge, 2012. p. 569-577.

SIMON, H. A. La sciences des systèmes: Science de l'artificiel. Paris: Epi, 1974.

STEINHART, E. Teilhard de Chardin and transhumanism. Journal of Evolution and Technology, Hartford, v. 20, issue 1, p. 1-22, 2008.

TEILHARD DE CHARDIN, P. L'Avenir de l'homme. Paris: Seuil, 1959. . L'Activation de l'énergie. Paris: Seuil, 1963. .TEILHARD DE CHARDIN, P., Science et Christ. Paris : Seuil, 1965.

TESTART, J.; ROUSSEAUX, A. Au péril de l'humain: les promesses suicidaires des transhumanistes. Paris: Seuil, 2018. (Science Ouvert).

TURNER, R. (Ed.). Transhumanism and Transcendence: Christian Hope in an Age of Technological Enhancement. Washington, D.C.: Georgetown University Press. 2011. p. 19-36.

Article soumis à l'évaluation le 13.09.2018 et approuvé le 30.11.2018.

François Euvé sj est docteur en théologie (Centre Sèvres, Paris), Professeur de théologie systématique au Centre Sèvres. Orcid.org/0000-0002-3831-8896

Courriel: francois.euve@centresevres.com

Adresse: 35bis rue de Sèvres 75006 Paris, France 